\title{
An attempt to scale subliminal visual stimuli'
}

\author{
A. G. Worthington \\ QUEEN'S UNIVERSITY, CANADA
}

\begin{abstract}
A scale of 'emotionality' was established for eight words. These eight words were then used in two separate sub-experiments. In the first, pairs of the words were shown and Ss were required to make brightness judgments. These judgments were not scalable. The same eight words were then used in a typical perceptual defense experiment. Perceptual defense and vigilance were observed.
\end{abstract}

\section{Problem}

Many psychologists feel that the phenomenon of perceptual defense is essentially an artifact explainable in terms of differences in word frequency and word familiarity between the 'emotional' and neutral material. Others consider that conscious suppression might well account for perceptual defense reactions. Still others argue that selective response sets might be the crucial determinants of selective responsitivity. Recent reviews by Eriksen (1960) and Brown (1961) provide comprehensive information on the history and present status of perceptual defense and vigilance.

In the author's opinion conventional methods of research in this area cannot provide definitive findings regarding the status of the perceptual defense concept. Research of the past $15 \mathrm{yr}$. , if it has demonstrated nothing else, has certainly shown that it is exceedingly difficult to control all variables which might prove pertinent.

In the study reported here a somewhat novel procedure was employed. Instead of meticulously selecting stimuli-possibly unsuccessfully anyway-in an attempt to meet the many powerful objections to perceptual defense it was decided merely to devise two situations in which the objections would be equivalent in power but where variations in methodology might produce two different sets of results.

\section{Method}

Sixty-four male Ss were required to make paired comparison judgments in connection with 15 words. They were asked to nominate which member of a stimulus pair they considered the more 'emotionally threatening'. The responses were scaled following Torgerson's (1958) Class II procedure i.e. an attempt was made to produce an 'average' scale for the population under consideration. All appropriate controls in the form of randomization, counterbalancing, etc. were applied. The eight words 'virgin,' 'penis,' 'death,' 'swine,' 'decay,' 'press,' 'branch,' and 'bread' were found to be the 'best' items and were selected to be used in Phases 2 and 3 of the study. A goodness of fit test applied to the scale based on these eight items produced $a x^{2}$ of 27.06 (df, 21; N.S.) indicating that the scale was usable.
Phase 2 of the study involved an attempt to scale subjective brightness judgments of pairs of the above stimuli. These stimuli were presented two to a slide (28 combinations in all). The objective brightness of each member of a stimulus pair was exactly equal. Each slide was presented for a period of $2 \mathrm{sec}$. at a level of illumination $0.2 \log \mathrm{ft}$ lamberts below a previously obtained recognition threshold based on stimuli similar to those used in the scaling task. The descending method of limits was employed to obtain this threshold estimate. Criterion of threshold was the lowest illumination at which Ss recognized either or both of the words in the test slides. Each $\mathrm{S}$ was required to make 112 judgments as to which of the two stimuli appeared the brighter. Order and position effects for the judgments were controlled by counterbalancing procedures. The 112 trials thus included four groups of the 28 independent stimulus pairs. Sixteen male Ss were used in this part of the study. Scaling was executed using the standard procedure for a mixed model (between and within Ss) - see Torgerson (1958, p. 175 ff).

In Phase 3 of this study an additional 16 male Ss were used. The eight stimuli employed in the scaling task were presented in counterbalanced random orders and both awareness and recognition thresholds were obtained. The ascending method of limits was employed.

\section{Results}

There was no evidence whatsoever that the brightness judgments obtained in Phase 2 were scalable. Judgments were almost completely random. In Phase 3, however, significant differences between words were observed at both awareness and recognition threshold levels (F, 2.47; df, 7/105; p < 0.05; and F, 7.02; df, 7/105; p < 0.001 respectively). At the awareness level there was quite clear evidence of perceptual defense, the words judged more 'emotional' in Phase 1 producing higher thresholds. At the recognition level a similar result was observed except that a vigilance reaction was noted to 'virgin,' the most 'emotional' word of the stimulus array. Indeed the recognition threshold to this stimulus was lower than the threshold of any of the 'unemotional' words.

\section{Diseussion}

Implicit in the design of this study was the assumption that a relationship should exist between threshold estimates and perceived brightness. If one stimulus has a higher threshold than another then it should be perceived as duller than the second and vice versa. If one grants the possible validity of this assumption then it becomes immediately apparent that in this study we have two independent methods of investigating the same 
phenomenon. The problem then becomes one of explaining the completely different results obtained in the two sub-experiments. It should be pointed out that any argument presented here is extremely tentative.

The most interesting point about these results is that although all the objections to an 'arousal' type of explanation of perceptual defense are equally relevant in both sub-experiments the phenomenon was observed to occur in only the second situation, i.e. conventional threshold measurement. Perhaps the most significant difference in the procedures lies in the fact that in the first experiment the Ss were always required to make a choice between two simultaneously presented stimuli while in the second experiment the judgment (or threshold) was obtained for each individual stimulus. It can be argued that the results obtained lend support to an 'arousal' type of explanation of perceptual defense similar to that advanced by Dixon (1960) and by Samuels (1959). If changes in 'arousal' are responsible for perceptual defense then in the paired comparison situation used here one would not expect to observe a scale since the change would not be stimulu-specific and although changes might occur with each slide presented Ss would be guessing within any given stimulus pair. In the second experiment, however, any change in 'arousal' would be stimulus-specific and hence the obtained results would be predicted.

Quite clearly a lot more work needs to be done before the argument advanced above can be considered powerful. It is proposed to extend the paired comparisons situation to the scaling of simultaneous and successive brightness judgments where every detail of the procedure will be identical except for the specific condition under which the judgment is made. If a scale is obtainable under the successive condition but not under the simultaneous condition then an 'arousal' type explanation would seem warranted.

\section{Referenees}

BROWN, W. P. Conceptions of perceptual defence. Brit. J. Psychol., 1961, Monograph supplement XXXV. DIXON, N. F. Apparent changes in the visual threshold: central or peripheral? Brit.J. Psychol., 1960, 51, 297-309.

ERIKSEN, C. W. Discrimination and learning without awareness: a methodological survey and evaluation. Psychol. Rev., 1960, 67, 279-300.

SAMUELS, I. Reticular mechanisms and behavior. Psychol. Bull., 1959, 56, 1-25.

TORGERSON, W. S. Theory and methods of s caling. New York: Wiley, 1958.

\section{Note}

1. This study was conducted as a part of a research program undertaken for the Ph.D. degree in the University of London. The author is indebted to the University of Queensland for financial support while this program was carried out. 